\title{
Ethnografie und Aufmerksamkeit Zur phänomenologischen Perspektivierung der Feldforschung
}

\author{
L. Frers \\ Telemark University College, Kjølnes Ring 56, 3918 Porsgrunn, Norway \\ Correspondence to: L. Frers (lars.frers@fu-berlin.de)
}

\begin{abstract}
Zusammenfassung. This article discusses how phenomenological procedures can be used to instruct ethnographic research. Since doing ethnography in the field builds on sensory perception, a closer inspection of relevant processes of perception is necessary. Insights into the field that is studied are dependent on the manner in which it is perceived. As Waldenfels" "phenomenology of attention" displays, the distribution of attention is of crucial importance in this context. To get to grips with the uses of phenomenology in the field, three areas of tension are explored: proximity - distance, mixture - analysis and presence - recording. These terminological pairs relate to tensions that structure experience and the play of perceptions in the field. Based on a reflection of these relations, the article offers a methodology that is characterized by mobility, openness and a continuous highlighting of how the researcher's corporal embeddedness into the field affects the distribution of attention.
\end{abstract}

1

Die Absicht dieses Artikels ist es jenen, die ethnografisch arbeiten bzw. arbeiten wollen, theoretisch fundierte Überlegungen mitzugeben, die ihnen sowohl bei ihrer Arbeit im Feld als auch bei der anschließenden Auswertung und Darlegung behilflich sind. Die Strategie dieses Artikels folgt dabei der Überlegung, dass praktisch sinnvolle Unterstützung weder über rezepthafte Anleitungen und standardisiertes Vorgehen noch über theoretische Orthodoxie $\mathrm{zu}$ erreichen ist. Es gilt vielmehr, theoretische Erwägungen so aufzubereiten, dass sie einen Bezug zu den unberechenbaren Herausforderungen bei der Arbeit im Feld aufweisen. So soll es möglich werden, dem Geschehen auf eine Weise zu begegnen, die sowohl direkt vor Ort einen zielführenden und gleichzeitig offenen Umgang mit den beobachteten Prozessen zulässt, die aber auch einen über das lokale Geschehen hinaus gehenden analytischen Zugriff ermöglicht. Diese Spannung zwischen Gemenge und Analyse, zwischen Eintauchen und Differenzieren soll hier im Hinblick auf die Rolle der Aufmerksamkeit fruchtbar gemacht werden. In dieser Weise wird der Komplexität des konkreten Geschehens Raum gelassen und gleichzeitig gezeigt, in welcher Weise methodisch gesteuerte Zugriffe auf das Geschehen möglich werden.
Eine solche Reflexion der Methode fordert auch die Geographica Helvetica. In ihrem Leitfaden für Forschungsartikel unter Punkt 2.3 verlangt sie für jeden eingereichten Artikel ein eigenes Kapitel zur Methode, in dem nachgewiesen werden soll, welche Methoden den im folgenden Abschnitt kommenden Resultaten zugrunde liegen. Für Standardmethoden reiche ein kurzer Hinweis, bei anderen Methoden könne auf entsprechende Quellen verwiesen werden und ,[b]ei spezielleren Methoden sollte ein leicht verständlicher Einblick sowie ein relevanter Quellenverweis gegeben werden.“ (Schneider-Sliwa et al., 2009:1).

Allem Anschein nach handelt es sich bei der in diesem Band der Geographica Helvetica im Mittelpunkt stehenden Ethnografie um eben eine solche ,speziellere Methode“. Es handelt sich hier nicht um eine Forschungsform, die es leicht machen würde, eine Hypothese aufzustellen, kontrolliert ermittelte empirische Ergebnisse zu präsentieren und im nächsten Schritt deren mögliche theoretische Implikationen zu diskutieren. Trotzdem hat die Ethnografie den Anspruch, mehr zu produzieren als reine Beschreibungen, in denen bloß ein lokales Geschehen detailliert geschildert wird. In der Ethnografie steht die Suche nach Zusammenhängen, nach neuen oder anderen Handlungs- bzw. Wahrnehmungsmustern im Vordergrund. Sie will verstehen, wie Zusammenhänge in der 
Praxis hergestellt werden. Dabei muss sie offen sein für das, was ihr begegnet.

All das heißt aber nicht, dass in der Ethnografie keine methodologische oder methodische Strenge gefordert wäre - ganz im Gegenteil. Die Forschungsarbeit, auf der dieser Artikel basiert, ist aus der Überzeugung hervorgegangen, dass sich in dem Umgang mit sozialwissenschaftlichen Methoden eine Nachlässigkeit eingeschlichen hat, die sich mit dem Mantel der wissenschaftlichen Normalität tarnt. In dieser Normalität wird methodologische Strenge als rein technische Anforderung gesehen, nach der bestimmte Verfahrensregeln eingehalten werden müssen. Regeln, die am besten in Instrumenten oder Algorithmen verfestigt sind. Wenn es gut läuft, werden dieser Logik nach beispielsweise die für die jeweils erhobenen Daten passenden Skalen berücksichtigt und aus diesem Grund die passenden statistischen Verfahren verwendet, oder - im Rahmen der qualitativen Forschung - die richtigen Kodierungsverfahren eingesetzt. Hier jedoch soll es darum gehen, das methodische Vorgehen über diese technischen Verfahren hinaus zu hinterfragen. Der Erfahrungskomplex, in dem und mit dem sich materielle genauso wie soziale Tatsachen überhaupt erst zeigen, soll methodischer Reflexion unterworfen werden. Dazu ist ein theoretisch-methodologischer Rückgriff auf die Phänomenologie nötig.

\section{Phänomenologie im Feld}

Die Phänomenologie ist für die hier präsentierte Perspektivierung der Ethnografie aus zwei Gründen richtungsweisend. Erstens drückt sie mit dem Motto „Wir wollen auf die ,Sachen selbst“ zurückgehen“ (Husserl, 1984, Bd. 1:10) die Motivation dieses Artikels treffend aus: den Willen sich den wissenschaftlichen Zugang zur sozial-räumlich-materiellen Welt nicht von etablierten Erwartungen einschränken zu lassen, sondern die zu untersuchenden Entitäten bzw. Prozesse selbst zum Ausgangspunkt der Untersuchung zu machen. Soll zum Beispiel die Rolle von Dingen im sozialen Handeln erkundet werden, dann reicht es nicht, sich nur mit dem Reden und Schreiben über die Dinge, also mit ihrer sprachlichen Konstruiertheit zu befassen. Man muss sich auch vor Ort dem praktisch-materiellen Umgang mit den Dingen aussetzen. Noch mehr als das, man muss verfolgen, wie die Dinge überhaupt erst zu dem werden, als das sie genommen werden.

Zweitens stützt sich ethnografische Forschung notwendigerweise auf die sinnliche Wahrnehmung der Forschenden, wobei diese sinnliche Wahrnehmung häufig mit einem visuellen bias als teilnehmende Beobachtung gefasst wird (Burawoy et al., 1991; Hammersley und Atkinson, 2007), gelegentlich aber auch explizit die anderen Sinne mit einbezieht (Degen, 2008 liefert ein schönes Gegenbeispiel). Die Untersuchung der Wahrnehmung wiederum ist eines der zentralen Felder, mit denen sich die Phänomenologie beschäftigt, insbesondere wenn sie, wie in diesem Beitrag, wie hier an Merleau-Ponty anknüpft.

Entsprechend der Zielsetzung dieses Beitrags wird das Wahrnehmen hier nicht als Kategorie oder eigene Entität als Wahrnehmung -, sondern als praktische Aktivität - als Wahrnehmen -, behandelt und so in seiner Rolle in jedwedem ethnografischen Arbeiten untersucht. Im Wahrnehmen konstituiert sich das untersuchte Geschehen und zwar im Zusammenhang von Geschehen und Beobachten. Selbst im visuellen Beobachten bindet das Geschehen diejenigen, die beobachten, körperlich ein. Sie müssen sich auf das zu Betrachtende hin orientieren, ihre Körperhaltung anpassen, ihre Augen- und Gesichtsmuskulatur beständig kontrollieren etc. Selbst wenn sie entfernt sind, sind sie nicht von dem Geschehen gelöst. Es beansprucht sie, sonst würde es für sie nicht wirklich werden. So sind Ethnografen wie andere Forscher auch immer Teil des Feldes, keine von ihm getrennten Subjekte, keine Hirne im Tank, die von außen über Nervenkabel mit Informationen über Dinge versorgt werden, die sie eigentlich schon kennen. Merleau-Ponty schreibt hierzu:

Die Welt ist kein Gegenstand, dessen Konstitutionsgesetz sich zum voraus in meinem Besitz befände, jedoch das natürliche Feld und Milieu all meines Denkens und aller ausdrücklichen Wahrnehmung. Die Wahrheit „bewohnt“ nicht bloß den ,inneren Menschen“, vielmehr es gibt keinen inneren Menschen: der Mensch ist zur Welt, er kennt sich allein in der Welt (Merleau-Ponty, 1974:7).

Auch wenn das Wahrnehmen in der ethnografischen Forschung in der Regel ein Beobachten ist, so stellt es sich aus phänomenologischer Perspektive nie als eine unkörperliche, rein rationale Tätigkeit dar. Alles Wahrnehmen ist körperlich gebunden oder besser gesagt: es ist Teil des leiblichen Bezugs zur Welt.

Hier treffen wir auf die erste Schwierigkeit im Umgang mit dem Vokabular der Phänomenologie. Wieso wird von Leib gesprochen? Dem Begriff wohnt im Alltagsgebrauch eine quasi-religiöse Konnotation inne, die ihn antiquiert erscheinen lassen mag. Mit dem Begriff wird aber eine wichtige Differenzierung vorgenommen: die zwischen Körper und Leib. Der Körperbegriff steht in diesem Zusammenhang für einen naturwissenschaftlich objektivierenden Zugriff, in dem der Körper zu einem Gegenstand der Erkenntnis reduziert wird. Er kann als solcher untersucht, analysiert und dann in einzelnen Teilen wieder zusammengesetzt werden. Der Leibbegriff hingegen steht für ein Verständnis, in dem der ständige und grundlegende innere Zusammenhang von Sinnen, Welt und Erkennen betont wird. Der Leib wird nicht wie der Körper als vom Geist getrennt gedacht, so wie das Subjekt vom Objekt getrennt ist, sondern als mit Dingen und Ideen immer schon verschränkt (Merleau-Ponty, 1974:75-79, 107114). Der Leib ist somit nicht wie der Körper bloßer Gegenstand der Wissenschaft, sondern der körperlich-sinnliche Ausgangspunkt aller Erfahrung. Damit ist er eben auch Ausgangspunkt aller Wissenschaft, die sich aus Wahrnehmungen und Erfahrung speist. 
Diese Qualität des Wahrnehmens, seine leibliche Gebundenheit, macht es zu dem Forschungsinstrument überhaupt es ist notwendigerweise immer Teil der Welt. Wie in methodischen Erläuterungen zur sogenannten teilnehmenden Beobachtung (DeLyser, 2010; Denzin und Lincoln, 2005) immer wieder betont wird: ohne Teilnahme gäbe es keinen $\mathrm{Zu}$ griff auf die Qualitäten und die Struktur des Geschehens. Die Teilnahme, als ein zur Logik des Geschehens Gehören, ermöglicht es erst, die Welt auch zu begreifen. An dieser Stelle tritt die zweite Schwierigkeit im Umgang mit der Phänomenologie zu Tage: Der Anspruch ist, dass ein Erkennen der Differenz von Körper und Leib und damit auch das Erkennen der Vorrangigkeit der leiblichen Erschließung der Welt dazu führen muss, dass ein Arbeiten auf phänomenologischer Grundlage quer zum „,normalen“ wissenschaftlichen Arbeiten verläuft, da dieses sich auf Grundlage überholter cartesianischer Unterscheidungen (Subjekt vs. Objekt) abspielt. Entweder man folgt den Prinzipien phänomenologischer Forschung (und begibt sich damit wahrscheinlich in eine weitgehende Isolation vom wissenschaftlichen Mainstream) oder man folgt den Kriterien eines rationalistischen Forschungsparadigmas (und ignoriert damit die zentrale Rolle der Leiblichkeit). Glücklicherweise zeichnet sich ein erheblicher Teil phänomenologisch arbeitender Forschung dadurch aus, dass sie offen für klassische wissenschaftliche Forschung ist und mit dieser gemeinsam an Problemen arbeitet - eine Offenheit die sich beispielsweise auch in den vielfältigen Bezügen zeigt, die Merleau-Ponty zur klinischen Psychologie herstellt. Gleichzeitig erfahren phänomenologische Untersuchungen auch in neueren Ansätzen der Neuropsychologie zunehmende Bestätigung, wie beispielsweise in Experimenten zur Wahrnehmung von Phantomgliedern (Merleau-Ponty, 1974:100-101; Guterstam et al., 2011; Ramachandran und Brang, 2009).

Im Folgenden wird es entsprechend darum gehen, die leibliche Verflechtung der ethnografisch Forschenden mit der Welt so zu hinterfragen, dass deutlich wird, wie sich die Forschenden selbst den Zugang zu Phänomenen verstellen. Grundsätzlich steht dabei im Vordergrund, dass sich das Wahrnehmen der Forschenden selbst vergessen macht, es also in der Regel gar nicht im Forschungsprozess berücksichtigt wird. Die soziale Einbindung der Forscherin im Feld ist ein klassischer Gegenstand sozialwissenschaftlicher Methodologie (Hirschauer und Amann, 1997). Die sinnliche Einbindung hingegen erscheint als weitgehende Leerstelle. Warum ist das problematisch? Zum einen sollte sich jedwedes solide methodische Verfahren sowieso darüber klar sein, was es erfasst und was es nicht erfasst. Zum anderen wird der blinde Fleck in Bezug auf das Wahrnehmen insbesondere dann relevant, wenn es um Effekte oder Zusammenhänge geht, die sich im Umgang mit der materiellen Welt konstituieren. Durch die zunehmende Beschäftigung der Anthropobzw. Kulturgeografie mit Fragen der Materialität (Kazig und Weichhart, 2009) werden diese sinnlichen Bezüge besonders relevant, wie insbesondere Forschungen zum Konzept der
Atmosphäre verdeutlichen (Hasse, 2008; Kazig, 2007). So stammen auch die folgenden Beispiele aus Forschungsvorhaben, in denen es eben darum geht zu zeigen, wie soziale bzw. kulturelle Prozesse über die Materialität der Welt konfiguriert werden. Die Crux dieser Konfigurationen liegt für die Forschenden genauso wie für die situationell Betroffenen darin, dass sie unbemerkt geschehen, weil sie sich im Wahrnehmen selbst herstellen. So wird die genauere Untersuchung des Wahrnehmens zu einem zentralen Aspekt der eigentlichen Forschung.

Diesem Problem wird in der Phänomenologie in der Regel durch die sogenannte Epoché begegnet, durch das gezielte außer Spiel setzen bestimmter Vorannahmen, die den Blick auf das zu untersuchende Phänomen also solches beeinträchtigen können (Vgl. Zahavi, 2007:21-25 für einen Überblick über verschiedene Spielarten der Epoché). In diesem Beitrag wird mit Hilfe der Epoché versucht, die Rolle der Aufmerksamkeit in der Feldforschung zu verstehen: wie richtet sich die forschende Aufmerksamkeit aus, was tritt aus dem Feld hervor und beansprucht Aufmerksamkeit, was wird in den Blick genommen?

\section{Aufmerksamkeitsgeschehen}

Die herausragende Rolle der Aufmerksamkeit wird zwar schon von Merleau-Ponty bedacht (Merleau-Ponty, 1974:4753), Waldenfels kommt aber das Verdienst zu, die Aufmerksamkeit systematisch als eigenes Phänomen zu untersuchen und in ihrer spezifischen Qualität darzustellen (Waldenfels, 2004). Waldenfels' Anliegen ist es, deutlich zu machen, dass es in der Aufmerksamkeit zwar einen Spannungsbogen zwischen dem gezielten Aufmerken auf etwas und dem zufälligen Auffallen von etwas gibt, dass das Aufmerksamkeitsgeschehen selbst aber nie der einen oder anderen Seite angehört. Im Aufmerksamkeitsgeschehen interagieren eben nicht zwei getrennte Entitäten (z.B. die Suchenden und das zu Findende) über bestimmte Akte, beispielsweise vermittels einer bestimmten Rationalität oder Handlungslogik. Es gibt auch kein Subjekt, das Objekte als solche überhaupt erst konstituiert und erkennt. Stattdessen ist das Aufmerksamkeitsgeschehen durch eine ständige Bewegung charakterisiert, in der vorher nie klar ist, was hervortritt bzw. entgegengenommen wird. Gleichzeitig ist das Geschehen auch nie abgeschlossen. Jedwede Konstitution von etwas im Feld der Aufmerksamkeit ist ihrerseits wieder einer steten Veränderung unterworfen, da sich die Kräfte oder Gewichte, die das Aufmerksamkeitsgeschehen prägen, ständig verlagern. Waldenfels verwendet die Länge eines gesamten Buches darauf, zu verdeutlichen, dass dieses Geschehen in jederlei Hinsicht offen und unstabil ist und das weder ein Subjekt über das bestimmt, was ihm entgegentritt, noch ein Objekt darüber, wie es gesehen wird. So schreibt er in Bezug auf den Übergang vom Aufmerken auf etwas zum Bemerken von etwas: 


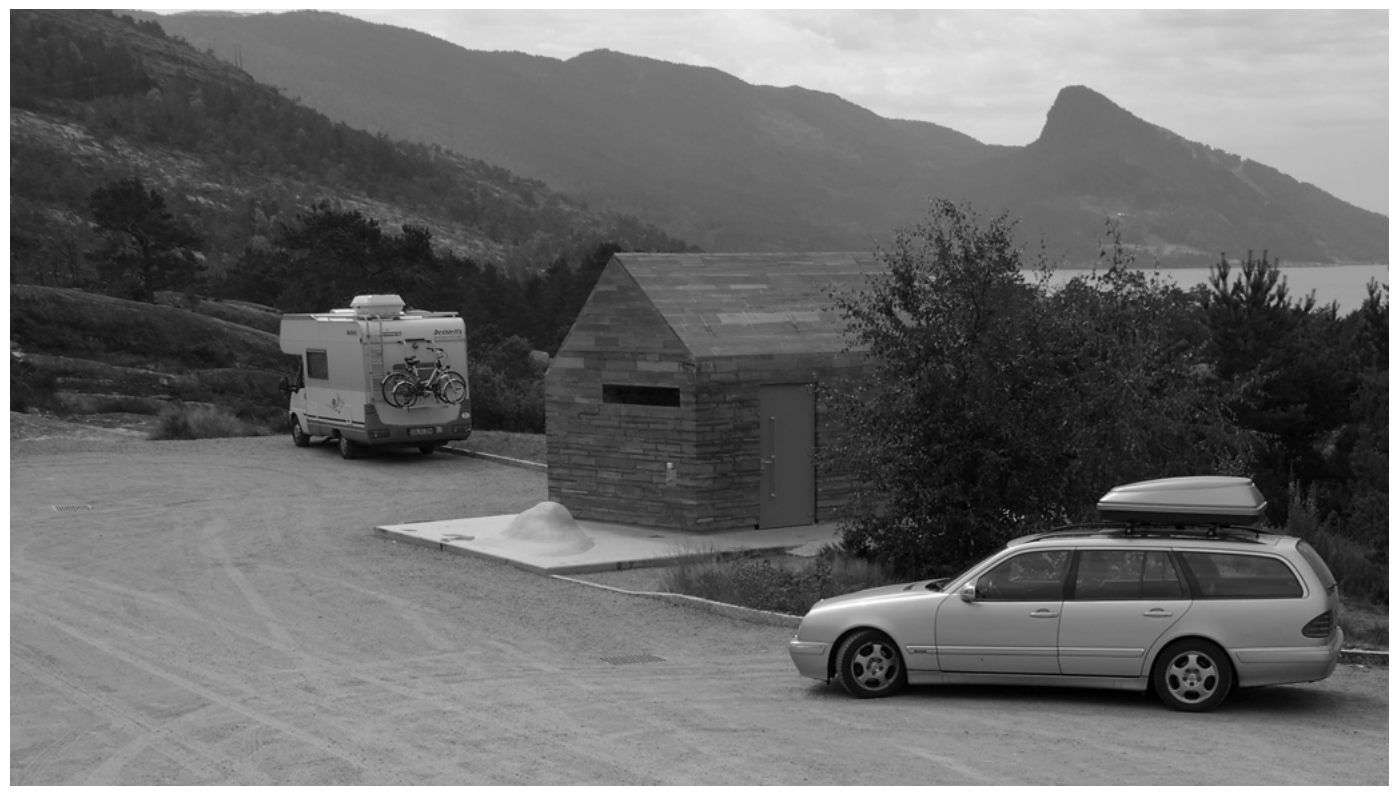

Abbildung 1. Gelbes Toilettengebäude in Hereiane, Lars Frers, Creative Commons by-nc-sa 3.0.

„Speziell geht es um die Frage, warum gerade dieses auftritt und nicht vielmehr anderes. Die zentrale Funktion, die von der Aufmerksamkeit an dieser Stelle übernommen wird, ist die der Reliefbildung. William James [spricht] von Selektion, wie man es heute vielfach tut, indem man mit Filtern und Rastern operiert. Doch diese Redeweise ist höchst mißverständlich. Sie erweckt den Eindruck als würde lediglich einiges ausgesondert, anderes weggelassen, einiges eingelassen, anderes ausgesperrt und als bestünde das Aufmerksamkeitsfeld aus einem Repertoire katalogisierter Daten, aus dem man sich je nach Bedarf bedient. Doch eine Aufmerksamkeit, in der die Erfahrung sich organisiert, in der Neues entsteht [...] leistet mehr und anderes. Sie sorgt dafür, daß in der Ausbildung eines ,Beachtungsreliefs' einiges hervortritt, anderes zurücktritt [...]. Dem entspricht auf seiten des Aufmerkenden ein Vorziehen und Zurückstellen. Auf diese Weise wird etwas zum Thema." (Waldenfels, 2004:101f)

In dieser Passage wird betont, dass es eine Doppelbewegung ist, die überhaupt erst die Entstehung von etwas Neuem ermöglicht. Hier kommen wir zum eigentlich Interessanten, zum Kern der Waldenfels'schen Kritik: Das traditionelle Verständnis der Aufmerksamkeit - als einer Art innerer Scheinwerfer, der gesuchte Dinge ins Licht rückt - weist Ähnlichkeiten zu einem Filter auf, der Bekanntes einordnet und zuteilt. Man könnte so beispielsweise die Beobachtung von bestimmten Handlungskategorien im Feld beschreiben. In der Aufmerksamkeit geht es aber eben nicht um ein solches Katalogisieren bekannter Größen. Es geht um das Ent- stehen von Neuem, um die Herausbildung eines Themas. Um dieses zu erfassen, müssen notwendigerweise beide „Seiten“ berücksichtigt werden, die Seite der Wahrnehmenden und die des sich Zeigenden.

So hat sich beispielsweise für mich im Rahmen eines Forschungsprojekts zur Norwegischen Tourismusroute die Frage ergeben, welche Rolle die Architektur der zur Tourismusroute gehörenden Rastplätze im Zusammenhang mit der Wahrnehmung norwegischer Landschaft spielt (Frers, 2011). Das in Abbildung 1 gezeigte Toilettengebäude ist ein Beispiel für die von Waldenfels beschriebene Qualität des Aufmerksamkeitsgeschehens. Man könnte sagen, dass das auf gelbem Grund stehende Toilettengebäude sich in das Wahrnehmungsfeld derjenigen drängt, die am Rastplatz vorbeifahren. Gleichzeitig ist es aber so, dass die Erwartungshaltung der fahrenden Person, die aktuelle Fahrpraxis usw. genauso den Prozess mitformen, in dem das Toilettengebäude als knallgelbes! komisches! Toilettengebäude erst entsteht und so die Fahrenden zum Anhalten veranlasst. Solche Prozesse, in denen nicht einfach etwas fertig gegebenes wahrgenommen wird, sind unter anderem zum Verständnis der Rolle der materiellen Welt in sozialen Prozessen von entscheidender Bedeutung.

Dabei gilt es zu beachten, dass eine Verhandlung der eigentlichen Phänomenologie der Aufmerksamkeit im Zusammenhang mit forschungspraktischen Fragen nicht ohne Reibung abläuft. Deshalb möchte ich im Folgenden drei Begriffspaare vorstellen, in denen auf der einen Seite eine phänomenologische Perspektivierung im Vordergrund steht, der auf der anderen Seite klassische Konzepte sozialwissenschaftlicher Methodologie gegenüber gestellt werden. Die Absicht hierbei ist nicht, grundsätzlich unterschiedliche 


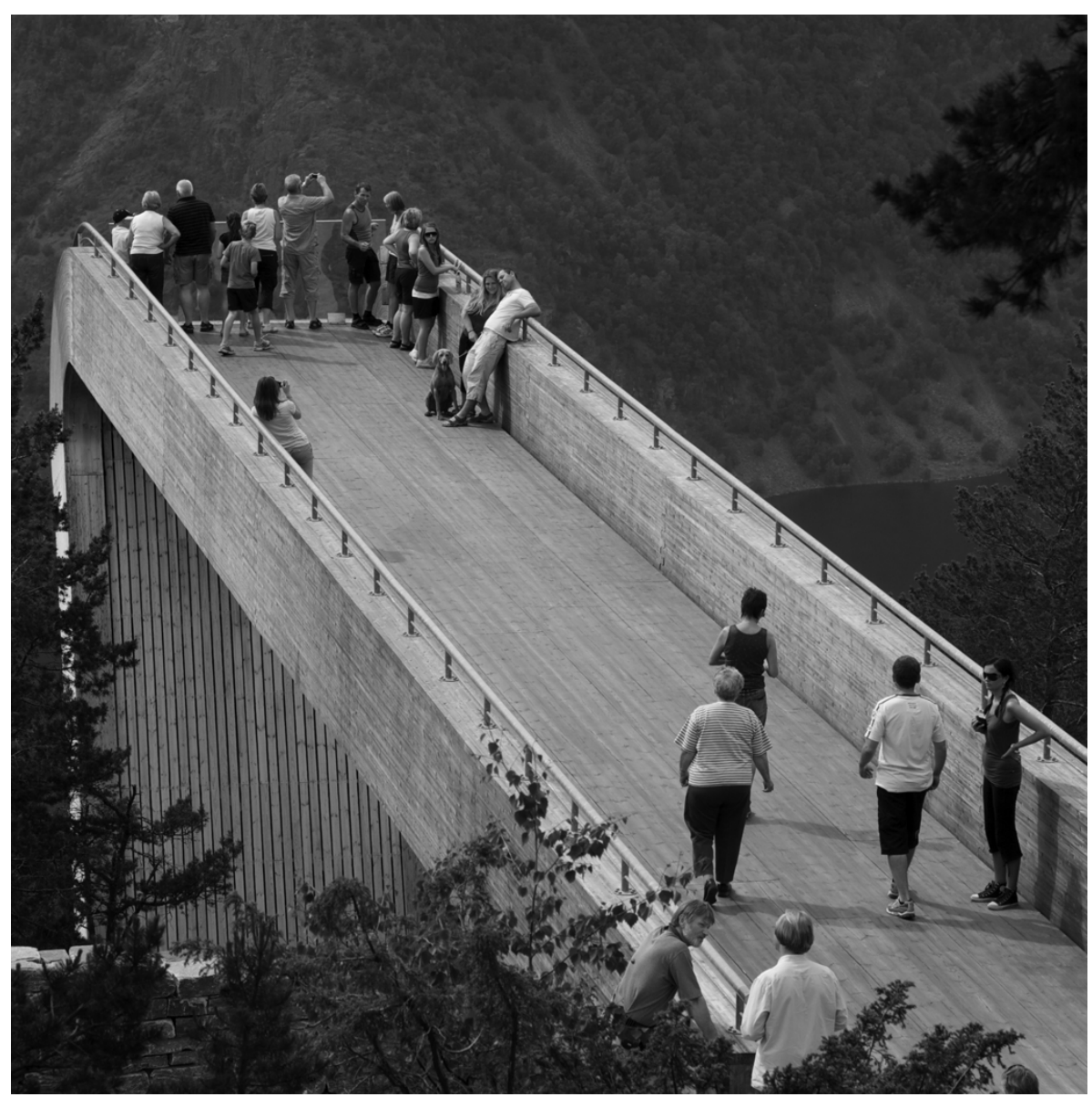

Abbildung 2. Stegastein Aussichtsplattform, Kerstin Bornholdt, Creative Commons by-nc-sa 3.0.

epistemologische Ansätze zusammenzuwürfeln oder als bloße Glosse zu behandeln, sondern im Spannungsfeld dieser Begriffspaare Raum für einen produktiveren Zugang zum Geschehen im Feld zu öffnen - als Erinnerung daran, wie man dem Problem überbordender Eindrücke genauso wie dem Problem der Trivialität des Normalen vor Ort begegnen kann.

Auf Abbildung 2 zeigt sich ein vielfältiges Getümmel: unterschiedliche Menschen, die zum Teil stehen, zum Teil gehen, ein Hund ist auch dabei, eine hölzerne Plattform, die über einen Fjord hinausragt, Menschen die posieren und fotografieren, Bäume, Geländer und vieles mehr. Das Foto wiederum ist aus einer etwas unklaren Distanz aufgenommen worden, es ermöglicht so, einen Überblick über das Getümmel zu bekommen. In der ethnografischen Forschung gibt es solche Momente der Distanz zum beobachteten Geschehen. Die Distanz erlaubt es, das Geschehen zu ordnen und zu kategorisieren. Ich selbst als Ethnograf befinde mich aber, zu dem Zeitpunkt an dem das Foto gemacht wurde, im Geschehen, stehe am unteren Bildrand und unterhalte mich mit einem deutschen Touristen, der diesen Ort aufgesucht hat. Hier habe ich die Nähe zum Geschehen gesucht und nehme offen daran teil, wenn auch in einer Randposition. Denn nur in der Nähe zum Geschehen kann ich wahrnehmen, wie die extrem dynamische Umgebung sich entfaltet und so ein spezifisches Aufmerksamkeitsgeschehen hervorgebracht wird. Wer orientiert sich wohin, wie legt sich das Geflecht von Wind, Stimmen und Gerüchen um alle Anwesenden, wandelt sich, löst sich auf und wird weitergetragen? Der situationsabhängige Charakter des Umgangs beispielsweise mit der Scheibe am Ende der Plattform von Abbildung 2 wäre ohne eine solche Nähe zum Geschehen nicht zu erfassen. Doch ein ungebrochenes Verweilen in großer Nähe zum Beobachteten, eine ungestörte Teilnahme würde es andererseits kaum ermöglichen, diese auch zu erforschen und in Hinblick auf außer dem Geschehen liegende Fragen und Probleme zu untersuchen. So muss in der Feldforschung ein Wechseln von Nähe und Distanz statt finden, damit sich das Geschehen in seinen unterschiedlichen Qualitäten zeigen kann, wie Elias für die Sozialwissenschaften im Allgemeinen bereits in seiner Gegenüberstellung von Engagement und Distanzierung dargelegt hat (Elias, 1983:128). Unterschiedliche Größen, Mengen oder, allgemein gesagt, unterschiedliche Skalen treten in unterschiedlichen Distanzen hervor. 
Nähe und Distanz müssen dabei nicht nur räumlich verstanden werden. Das Wahrnehmen spielt sich auch in zeitlichen Dimensionen $\mathrm{ab}$, in denen das Geschehen andere Qualitäten gewinnt, wenn es aus einer anderen Zeit oder in einer anderen Geschwindigkeit betrachtet wird, wie zum Beispiel bei Videoaufnahmen möglich.

Das Getümmel von Abbildung 2 zeigt ebenfalls, wie ethnografisches Forschen Situationen hervorbringt, in denen Spannungen zwischen Teilnahme und Beobachtung entstehen, die den Gewichtsverlagerungen im Aufmerksamkeitsgeschehen entsprechen. So steht auf der einen Seite das Gemenge, in das hineingetaucht werden muss, um es als solches in seiner Eigenheit erfahren zu können. Wenn ich selbst auf der Plattform unterwegs bin, auf der Suche nach einer geeigneten Perspektive, aus der ich ein Foto schießen kann, das ich später auf Facebook präsentieren könnte, dann spüre ich, wie einen das Warten darauf quälen kann, dass andere endlich am Ende der Plattform Platz machen. Gleichzeitig bekomme ich mit, wie und worüber sich andere dort unterhalten und erhalte so Zugang zu ihren Beobachtungen und Erzählungen. Oder ich muss meine eigene Rolle als Mann auf der Plattform gemeinsam mit den Anderen aushandeln, mit einem Interviewpartner, der sich auf's Bauen versteht, mit einer attraktiven Frau und ihrem Partner, die sich entspannt über die Plattform bewegen. Ich bin mittendrin im Gemenge. Auf der anderen Seite ziehe ich mich manchmal zurück aus dem Gemenge, ordne es, indem ich es aufteile, zergliedere und die Teile zueinander in unterschiedliche Verhältnisse setze. Die Bewegung zwischen Gemenge und Analyse kennzeichnet das zweite Spannungsfeld, aus dem sich ein ethnografisches Forschen speist, das die Qualitäten des Wahrnehmens selbst mitberücksichtigt (Für eine ausgearbeitete „Philosophie der Gemenge" vgl. Serres, 1998).

Der Aufenthalt im Feld dient im klassischen Sinne der Erfassung des zu untersuchenden Zusammenhangs. Da dieser aber nicht einfach für sich gegeben ist, sondern als solcher, bzw. als Thema im von Waldenfels gemeinten Sinn, erst wahrgenommen und sich damit im Aufmerksamkeitsfeld realisieren muss, ist es einerseits notwendig diese Einbindung in ein dynamisches, sich ständig bewegendes Geschehen nachzuvollziehen - zu erfahren, wie mal etwas aufscheint, um nur kurze Zeit später wieder in den Hintergrund zu treten. Andererseits ist es im Forschungsprozess auch notwendig, das Wahrgenommene festzuhalten, bevor es sich verflüchtigt. So sind die Aufzeichnungstechniken und -technologien, Kodierungsverfahren und Logbücher wesentlicher Bestandteil ethnografischen Arbeitens im Kampf gegen das Vergessen und Verschwinden (Hammersley und Atkinson, 2007; Lofland et al., 2006). Ein Kampf, der immer auch schon gescheitert ist, da er die Eigenheit des Aufmerksamkeitsgeschehens nie einholen kann, wie Waldenfels im Zusammenhang mit dem Unerinnerbaren schreibt (Waldenfels, 2004:93).

Gleichzeitig wird die Erfahrung dieser Abwesenheit noch schmerzlicher, da selbst der Versuch des Festhaltens die ei- gene Einbindung in das gegebene Aufmerksamkeitsgeschehen von diesem abwendet und statt dessen zu einer Fokussierung auf das Festhalten führt - in diesem Fall das Protokollieren. Dies ist der dritte wichtige Aspekt, über den sich ethnografisches Forschen in der Praxis konstituiert: die Spannung zwischen Gegenwart und Aufzeichnung. Wann verlässt mein Wahrnehmen das Geschehen eines Gesprächs, um sich auf das Notieren einiger Stichworte zu konzentrieren? Wie wichtig ist es mir, die Dauer des Aufenthalts der einzelnen Gruppen vor Ort aufzuzeichnen, wenn das bedeutet, dass ich dann nicht mehr dabei sein kann, wie einzelne Mitglieder der Gruppe beim Verlassen des Ortes sich noch einmal umwenden, um einen letzten Blick auf den Fjord zu erhaschen? Die Benutzung von Ton-, Bild- und Video-Aufzeichnungsgeräten hilft dabei, solche Entscheidungen nicht so häufig treffen zu müssen. Gleichzeitig verändert die Nutzung eben dieser Technologien sowohl die Gegenwart im Feld als auch den späteren Zugang zum Geschehen. Mit Kamera sind weder ich noch die Anderen dieselben wie ohne Kamera (wobei dieses Anderssein nicht nur problematisch ist, sondern auch Chancen bietet, vgl. Frers, 2009).

Die Zusammenstellung der drei hier vorgestellten Spannungspaare - Nähe und Distanz, Gemenge und Analyse, Gegenwart und Aufzeichnung - erhebt keinen Anspruch auf Vollständigkeit. Das für die ethnografische Arbeit im Feld so entscheidende wie komplizierte Aufmerksamkeitsgeschehen ist eine permanente Herausforderung. Um dieser Herausforderung so entgegenzutreten, dass daraus eine produktive Begegnung wird in der etwas neues zu Tage tritt, muss klar sein, dass sich die Spannungen nie auflösen lassen. Es gibt keinen festen Ausweg. Das einzige was hilft, ist sich die Beweglichkeit zu bewahren, offen zu sein für Konstellationen, in denen von einem Modus zum anderen gewechselt werden kann.

\section{4 ,Mittendrin statt nur dabei“}

Distanzierte Beobachter, die messen und registrieren, eintragen und standardisieren, sehen vieles und vieles sehr genau (In der Tat stellt sich die Auswertung von gesammelten Daten selbst auch als ein Prozess dar, in dem Neues auf die gleiche Weise entsteht wie auch beim Aufmerksamkeitsgeschehen im Feld - allerdings mit anderen Gegenstandsbezügen). Sie sind jedoch nicht mittendrin im Geschehen, sie sind „nur dabei“ und haben keinen Zugang zur Entfaltung des Geschehens selbst. „Mittendrin statt nur dabei“ hier wurde dieses Motto so gewendet, dass es sich gewissermaßen auf das taktische Vorgehen im Feld bezieht. Die eigene Gegenwart im Gemenge, die Nähe zum Geschehen wurde hier distanzierten Bezügen gegenübergestellt, in denen Techniken des Aufzeichnens und der Analyse das Verhältnis zum Feld bestimmen. Dabei gilt es zweierlei zu beachten. Zum einen sollte deutlich geworden sein, dass das Aufgehen in der Situation allein nicht weiterhilft. Eine ständige Bewegung zwischen eintreten und sich entfernen muss, parallel 
zur Offenheit und Schließung des Geschehens den Forschenden gegenüber, aufrecht erhalten werden. Diese sich fortschreibende Spannung darf nicht aufgehoben werden, sonst würde das Forschen nur vorhandene Schemata reproduzieren. Dass das Einnehmen einer solchen Haltung oder besser gesagt das Aufrechterhalten einer solchen offenen Bewegung keinesfalls einfach ist, veranlasst Waldenfels dazu, seine Erläuterungen zur Phänomenologie der Aufmerksamkeit mit der folgenden, vorsichtigen Formulierung im Konjunktiv abzuschließen: ,, Die Schwebehaltung, die der Phänomenologe einnimmt, würde zulassen, daß sich die Gewichte der Dinge ändern und nicht nur ihre Gestalten.“ (Waldenfels, 2004:287)

Zum anderen sollte in diesem Artikel deutlich geworden sein, dass eine phänomenologischen Perspektivierung des eigenen Forschungsvorhabens auch dann gewinnbringend sein kann, wenn diese gewissermaßen nur in Ergänzung bestehender Herangehensweisen vorgenommen wird. Wenn sich die Phänomenologie als solche auch durch eine radikale Abkehr von etablierten, rationalistischen Wissenschaftsverständnissen auszeichnet, so heißt dies jedoch nicht, dass in einer Zeit der multiparadigmatischen sozialwissenschaftlichen Forschung (Gioia und Pitre, 1990; Terre Blanche und Durrheim, 2006) Kombinationen unterschiedlicher Forschungsperspektiven nicht möglich wären. Sollte die Mitberücksichtigung phänomenologischer Ansätze dazu führen, dass eine kritische Reflexion vorhandener epistemologischer Annahmen bezüglich des Status der Wahrnehmung bzw. der Aufmerksamkeit im Forschungsprozess stattfindet und so deutlich wird, dass das Auftreten von Zusammenhängen weder in den Dingen selbst noch im Cogito der Forschenden liegt, dann wäre dies sicher kein Verlust.

\section{Literatur}

Burawoy, M., Burton, A., Ferguson, A. A., Fox, K. J., Gamson, J., Hurst, L., Julius, N. G., Kurzman, C., Salzinger, L., Schiffman, J., und Ui, S. (Hrsg.): Ethnography unbound: Power and resistance in the modern metropolis, Berkeley, University of California Press, 1991.

Degen, M.: Sensing cities: Regenerating public life in Barcelona and Manchester, London, Routledge, 2008.

DeLyser, D. (Hrsg.): The SAGE handbook of qualitative geography, Los Angeles, SAGE, 2010.

Denzin, N. K. und Lincoln, Y. S. (Hrsg.): The SAGE handbook of qualitative research, 3rd Edn., Thousand Oaks, Sage Publications, 2005.

Elias, N.: Engagement und Distanzierung - Arbeiten zur Wissenssoziologie 1, Frankfurt am Main, 1983.

Frers, L.: Video research in the open: Encounters involving the researcher-camera, in: Video interaction analysis : Methods and methodology, Herausgeber: Tikvah Kissmann, U., Frankfurt am Main, 155-177, 2009.
Frers, L.: Stop, rest and digest: Feeding people into nature, in: Routes, roads and landscapes, Herausgeber: Hvattum, M., Brenna, B., Elvebakk, B., und Kampevold Larsen, J., Aldershot, 191200, 2011.

Gioia, D. A. und Pitre, E.: Multiparadigm perspectives on theory building, The Academy of Management Review, 15, 584-602, 1990.

Guterstam, A., Petkova, V. I., und Ehrsson, H. H.: The illusion of owning a third arm, PLoS ONE, 6, e17208, doi:10.1371/journal.pone.0017208, 2011.

Hammersley, M. und Atkinson, P.: Ethnography: Principles in practice, 3rd Edn., London, Routledge, 2007 [1983].

Hasse, J. (Hrsg.): Schwerpunkt: Stadt und Atmosphäre - Vierteljahreszeitschrift für Stadtgeschichte, Stadtsoziologie, Denkmalpflege und Stadtentwicklung, Remshalden, 35, 1-198, 2008.

Hirschauer, S. und Amann, K. (Hrsg.): Die Befremdung der eigenen Kultur: Zur ethnographischen Herausforderung soziologischer Empirie, Frankfurt am Main, Suhrkamp, 1997.

Husserl, E.: Logische Untersuchungen: Untersuchungen zur Phänomenologie und Theorie der Erkenntnis, Husserliana XIX, Den Haag, Martinus Nijhoff, 1984 [1901].

Kazig, R.: Atmosphären: Konzept für einen nicht repräsentationellen Zugang zum Raum, in: Kulturelle Geographien: Zur Beschäftigung mit Raum und Ort nach dem Cultural Turn. Kultur und soziale Praxis, Herausgeber: Berndt, C. und Pütz, R., Bielefeld, 167-187, 2007.

Kazig, R. und Weichhart, P.: Die Neuthematisierung der materiellen Welt in der Humangeographie, Berichte zur deutschen Landeskunde, 83, 109-128, 2009.

Lofland, J., Snow, D. A., Anderson, L., und Lofland, L. H.: Analyzing social settings: A guide to qualitative observation and analysis, 4th Edn., Belmont/CA, Wadsworth, 2006 [1984].

Merleau-Ponty, M.: Phänomenologie der Wahrnehmung, Translated by Boehm, R., Berlin, Walter de Gruyter \& Co., 1974 [1945].

Ramachandran, V. S. und Brang, D.: Sensations evoked in patients with amputation from watching an individual whose corresponding intact limb is being touched, Arch. Neurol., 66, 1281-1284, 2009.

Schneider-Sliwa, R., Korf, B., Reynard, E., und Mosimann, T.: Leitfaden für Forschungsartikel in der Zeitschrift Geographica Helvetica: Unsere Vorgaben und Bitten an Autorinnen und Autoren, http://www.geographicahelvetica.unibas.ch/pdf/guidelines dt.PDF, last access: 29 March 2011, 2009.

Serres, M.: Die fünf Sinne : Eine Philosophie der Gemenge und Gemische, Translated by Bischoff, M., Frankfurt am Main, Suhrkamp, 1998 [1985].

Terre Blanche, M. J. und Durrheim, K.: Histories of the present: Social science research in context, in: Research in practice: Applied methods for the social sciences, Herausgeber: Terre Blanche, M. J., Durrheim, K., und Painter, D., Cape Town, 1-17, 2006.

Waldenfels, B.: Phänomenologie der Aufmerksamkeit, Frankfurt am Main, Suhrkamp, 2004.

Zahavi, D.: Phänomenologie für Einsteiger, Paderborn, Fink, 2007. 